\title{
Randomised controlled trials: evaluating and communicating treatment effects
}

In the sixth paragraph of the answers section in this Endgames article (BMJ 2014;348:g1905, doi:10.1136/bmj.g1905), the authors wrote: "To have direct clinical relevance, the number needed to treat would be rounded to 22 patients," but they should have said 21 , not " 22 ," patients. 